\title{
Addressing the Mental Health Needs of Post-Secondary Students: Considerations for College Administration
}

\author{
Jordan M. Talley ${ }^{1 *}$
}

\begin{abstract}
Anxiety and depression are the leading causes for diminished performance and quality of life for students in their post-secondary academic career. The effects range from poor grades and dropping out to substance misuse and even suicide amongst members of student bodies nationwide. Students who are unaware of resources available to them are at risk of having these issues exacerbated over time. It is the responsibility of college administrations to prepare students for a life after college and, therefore, must enhance the treatment services and education of said services to the student bodies as a whole to alleviate these problems and to attempt to deter falling retention rates and potential tragedies on campuses.

\section{Keywords}

Student Mental Health — Anxiety and Depression — Substance Abuse — Student Success

${ }^{1}$ Integrative Studies, University of North Texas

*Faculty Mentor: Dr. Justin Watts
\end{abstract}

\begin{tabular}{lll}
\hline \multicolumn{2}{c}{ Contents } \\
\hline & Introduction & 1 \\
1 & Literature Review & 2 \\
2 & Future Directions & 5 \\
3 & Conclusion & 6 \\
& Author Biography & 6 \\
& References & 6
\end{tabular}

\section{Introduction}

Winerman states that the ratio of students to faculty in counseling centers is 1,737:1. This is an average. Smaller schools with 1,500 students or less had a ratio of 705:1, while larger institutions of 35,000 students or more had a ratio of 2,624:1.[1] This means that beyond the rising prevalence of mental health disorders on our campuses, universities are also under-equipped to handle monstrous caseloads and are rendered potentially ineffective in this battle. The problem here is not just one of the state of mental health of the student body but also one of resources available for those in need, such as counseling and other forms of care required for collegiate success for those suffering. This paper is intended to define the specific issues faced by post-secondary students with mental health issues and discuss plausible directions for addressing their needs by university administrations.

According to Amy Novotney, nearly half (45.2\%) of all visits to university counseling centers cited mental health related concerns as a primary issue. In 2013, they reached
48.7\%.[2] These numbers are startlingly high as they represent nearly half of collegiate populations represented by the studies conducted. Dastan Faeq states that the most common problem that is encountered by university counseling service centers is depression, representing over a quarter $(27 \%)$ of the symptoms expressed by students.[3] Novotney displays a graph depicting anxiety as being more prevalent than depression, while Faeq maintains that depression is the greater culprit. Regardless, both can agree that depression and anxiety are the two most common problems associated with the mental health issues faced by university students. Novotney cites a 2013 study done by National College Health Assessment that revealed that over 30 percent of those who sought services for mental health issues report that they have had serious considerations of attempting suicide at one time or another in their lives and about one-third of college students in the U.S. had problems functioning over the last 12 months because of their depression.

Anxiety is cited as the leading mental health concern facing post-secondary students reported by $61 \%$ of students visiting counseling centers on campus with depression cited at $49 \%$, according to the same article.[1] According to the DSM-V and the American Psychological Association, anxiety is the "anticipation of future threat," and the disorders consist of varying degrees of the responses to fear, anxiety, and the associated behaviors. Anxiety is split up between multiple disorders that "differ from one another in the types of objects or situations that induce fear, anxiety or avoidance behavior, and the associated cognitive ideation."[4] According to my 
research, whether or not the response to fear is appropriate or excessive must be made by a clinician. This occurs when the symptoms observed do not match or appear appropriate to the situation and are also not able to be addressed by the presence of other pre-existing mental health conditions or medications to which the symptoms can be attributed.

The nature of depression itself is extensive, according to the Diagnostic and Statistical Manual of Mental Disorders, 5th ed. (DSM-V), written by the American Psychological Association (APA). To simplify depressive disorders, the DSM-V (2013) states that the various expressions of depression are united by the presence of sad, empty or irritable moods that are coupled with somatic and cognitive changes that deeply affect a person's ability to function.[4, 155] It is also noted that those involved should be aware that normal sadness is not a depressive disorder and that bereavement, as an example, can induce suffering but does not indicate a depressive disorder. This is important as simple sadness for logical reasons does not constitute depression or mental disorder. For example, counseling may be sought for something as major as the loss of a loved one and this is common for a student to experience during their college career that can span anywhere from 2 years for an associate degree to 12 years depending on graduate programs and potential residencies.

One important consideration that must be made regarding the annual increase in students seeking treatment for mental health issues is simply that more students are enrolled and are attending college each year. This can explain some of the increases represented in the surveys but do not explain why these disorders are so prevalent. It also cannot excuse the self-injury reports. As far as non-suicidal attempting injuries went (i.e. pulling hair, cutting, burning), $21.8 \%$ of students in the survey participated in such behaviors in 2011-2012 and this number rose to $23.2 \%$ in 2012-2013. More serious is the number of students that seriously considered an attempt on their life. These numbers ranged from $23.8 \%$ in $2011-2012$ to $30.3 \%$ in 2012-2013. Alarmingly, in the surveys presented in Novotney's research, it is shown that the rise in suicide attempts of these students went from $7.9 \%$ to $8.8 \%$ from the 2010-2011 academic year to the 2012-2013 academic year.[2] Regardless of a rise in population overall on college campuses, these numbers are terrifying and represent a major problem that needs to be met with a more efficient means of treatment.

Depression and anxiety of this nature often lead to the misuse of substances such as alcohol and drugs, whether prescription or other. When considering the typical college lifestyle, drugs and alcohol are already widely available and are common in recreational use at parties and other student gatherings.

The DSM-V (2013) discusses this as substance/medicineinduced depressive disorder.[4, 177] According to the DSM-V (2013):

The diagnostic features of substance/medicationinduced depressive disorder include the symptoms of a depressive disorder, such as major depressive disorder; however, the depressive symp- toms are associated with the ingestion, injection, or inhalation of a substance (e.g., drug of abuse, toxin, psychotropic medication, other medication), and the depressive symptoms persist beyond the expected length of physiological effects, intoxication, or withdrawal period.

This is especially dangerous when mixed with depression, as many with depression do not understand their condition or even see that they have a condition at all. I do not intend to focus on substance misuse as a whole but as a symptom of depression and anxiety that may be capable of being noticed before one might realize the reality of the student's depression. Students who may not recognize their symptoms as depression may self-medicate with drugs, alcohol, and illegally/legally obtained prescription medication. Through this, not only can the symptoms of the depression itself be heightened to an out of control level, but there are various other severe complications that can arise from abuse of substances leading from disciplinary expulsion of the student from the institution to the death of the individual due to overdose or suicide.

\section{Literature Review}

Post-secondary students face a range of stress-inducing activities, from moving away from home, initiating real-world responsibilities (often for the first time in life), course workloads, set deadlines for projects, balancing work and social lives, and the drive to be successful and competitive amongst the class. Community colleges themselves are designed for students to commute to school within their home community and must balance working a job, sometimes multiple jobs, in order to make ends meet financially. Non-traditional students who no longer live with their parents, and are often parents themselves, balance potential child-care responsibilities, jobs, insurance and car payments, rent, and a potential lack of the aforementioned social life, which leads to loneliness that in itself can be stressful as well. An improper life balance can lead to heightened levels of stress that can cause or exacerbate issues of anxiety and depression, often leading to substance misuse disorders as the individual attempts to drown out the stress and emotions. Community colleges and universities must be a primary source for assistance to students throughout all of these potential issues faced during their time seeking degrees. International students have their own unique situations simply due to being from another nation altogether such as language barriers and a foreign education system. Sakurako Mori, in their article Addressing the Mental Health Concerns of International Students (2000), states that "given the evidence that the demands for cultural adjustments frequently place international students at greater risk for various psychological problems than are students in general, it is important that sufficient and readily accessible mental health services be established for them."[5]

The university that a student attends is a new world and culture that they enter into. For the students who are no longer 
at home with their parents and for those who have moved great distances to attend said universities, these institutions are basically their full-time home. Resources are available on campuses to students that cover a wide range of needs that students have during their attendance. Maslow's hierarchy shows the multiple levels of needs within each person that must be met for overall health of their being. This is broken into five levels in the shape of a pyramid, shown in Figure 1.

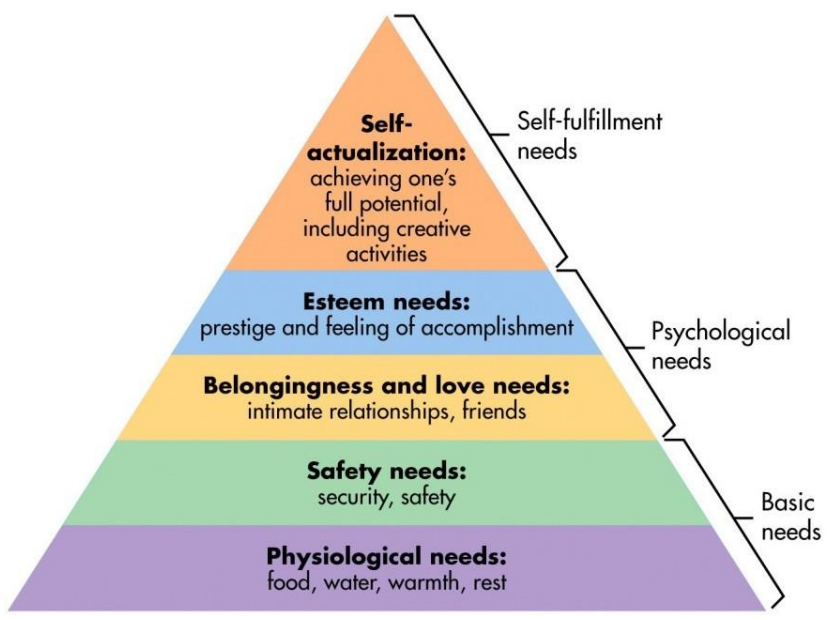

Figure 1. The Hierarchy of Needs

This paper focuses on the second level, safety needs. These represent "protection from elements, security, order, law, stability, and freedom from fear.'[6] Notice that these are shown to come after the base physiological needs such as food, shelter, warmth, and clothing are met, as those are strictly required for survival in general. This level also lies beneath the social needs which make up friendship, intimacy, family, and a sense of connection. This means that safety needs can only be met after survival needs and must be met themselves in order for relationships as a whole to develop in any capacity on the social level.

Shelter as a survival need is met through dormitories, usually paid for through student grants, loans or parents if the student is not working while attending school. Clothing is often brought from home, though clothing banks are available as a resource for those in need, just as food can be provided for students in need of a meal. The University of North Texas, for example, has a number of external and nearby resources for meals on certain days such as church groups next to the campus who offer lunch at no charge on Thursdays. Meal plans are available to students that can be fulfilled through various cafeterias and restaurants at a cost paid prior to the beginning of the school year that allot a certain number of meals per day. Food banks are present as well for those in serious need. Social needs are advertised by universities with fraternities, sororities, sporting events, classmate relationships, pep rallies, and local hangouts that all contribute to the overall "college experience." For social needs to be met within post-secondary students, security needs must be met first. Which begs the question: why are students slipping through the cracks when their security needs are concerned? If a student were unable to secure housing on a campus, they would most likely drop out of that institution, just as they would if they were unable to find a meal on a reliable and regular basis. Universities have answers for these items. Student activity groups and social gatherings are highly accessible and recommended to students who look for somewhere to belong. But when students have issues such as depression and anxiety, universities are far from equipped to address these issues. Why?

Universities are not intentionally letting this issue be perpetuated year after year. Colleges are at an immediate disadvantage for handling crises with students due to confidentiality laws that prohibit university staff from contacting family or care providers as well as the instance where symptoms experienced by students stop them from asking for help, thus leaving the students undiagnosed regarding their condition.[7] But there are numerous problems that could be handled on the university level. Winerman's findings on student-to-counselor ratios showed that a recent average was $1,737: 1 .[1]$ Stephen Bushong states that the IACS (International Association of Counseling Services) recommends a ratio of 1,500:1 studentsto-counselors. For larger universities, he cited an average of 2,607:1.[8] Since this was an article published in 2009, Winerman's more current findings show that things are getting better on the student-to-counselor ratios, but not by much. Furthermore, the mental health needs of students are increasing at an alarming rate, which means that these more current averages are made up of much higher percentages of students actually requiring mental health services. Therefore, even though the averages are getting slightly better, potential caseloads are higher and more students are not getting the help they require because universities lack the appropriate staff to handle them. The Texas Tribune is quoted as stating, "Understaffed counseling centers at Texas universities say they are frustrated by their inability to reach students, but the state doesn't earmark money for mental health services, and lawmakers want to limit tuition hikes. That leaves universities strapped for funds to hire more counselors and improve services."[9] Therefore, it is not only an issue of ratios but an issue of the financial needs of the universities in question that prohibit them from rectifying the current state of understaffed counseling centers.

The IACS, regarding the overwhelming ratios mentioned above, states five specific impacts that these numbers will potentially cause as they continue to rise. First and foremost, the wait-list for student seeking treatment will increase, leading students to simply not seek care at all or possibly leave the university altogether. The IACS cites a study performed by Wilson, Mason and Ewing from 1997 that "found that attrition rates increased by $14 \%$ for those students who were put on a wait list compared to those who received timely counseling." It also was stated that the liability of universities increases with wait list times.[10]

Second, the difficulty of providing quality care to the students who need attention for more serious situations increases. $95 \%$ of the 367 universities that were surveyed by the National 
Survey of Counseling Center Directors in 2013 claimed that the number of students with severe mental health related concerns had increased over the past few years.[10] This causes the amount of time needed to be spent with these students to increase for them to receive appropriate care. The IACS states that as severity increases, these ratios that have been mentioned should be decreasing so that students get the care they require. This is not the case, currently.

Third, which was mentioned briefly in the first item on the list, is the increased liability of colleges in the events that major tragedy occurs. These counseling centers have heightened liability when operating out of the recommended standards that have been set, i.e. a 1500:1 student-to-counselor ratio. The ratio at The University of North Texas, as of August 9, 2016, was 2,655:1, according to the Texas Tribune.[9] The IACS states that universities in this situation are "legally vulnerable.”[10]

Fourth, and possibly one of the most important when concerning higher education institution, is that as counseling services are stretched too thin, it is harder to support the academic success of the enrolled students. The percentage of students citing counseling as being a factor to their success in satisfaction surveys was $69 \%$. Counseling centers play a major role in aiding students along their journey through college but are being inhibited in their abilities when understaffed at this level.

Finally, as ratios of this nature increase, the counseling centers are unable to support the campus community (i.e. training faculty members, student group outreaches, consulting with faculty and staff on student related issues, etc.). The Virginia Tech shooting is mentioned here. After the shooting took place, counseling centers became responsible for training faculty and staff members at universities to recognize behaviors that might indicate a student could be a potential threat to themselves or others. School shootings are not a thing of the past. This training is more relevant than ever. However, clinical requirements per the increasing student demand for mental health services will prohibit counseling centers from handling this much needed task. Also, not all students who need help approach the counseling centers to receive the help they require, either due to symptoms they are experiencing or stigma that is associated.

When students approach these counseling centers, they are presented with many options. Traditionally, treatment takes the form of a short-term solution such as 6-8 weeks of weekly sessions. Campus-based counseling centers are not meant to be long-term when utilized. However, they are able to provide referrals to longer-term care via networks setup through Referral Specialists who may be present in the center that can aid students in making contact with those in the surrounding community. These people in external healthcare networks can take the student's treatment to the next level. One of the issues faced by many students in this instance is a lack of health insurance, especially present in non-traditional students who are no longer on their parent's insurance policy due to age-restrictions. Medication is another option that can be fulfilled through facilities like the Student Health and Wellness Center present on the University of North Texas campus.

Eisenberg highlights over some statistics based on his own findings. These record the percentages of students who received varying degrees of treatment from counseling centers. In his sample, $13.7 \%$ of students surveyed were receiving psychotropic medications, $14.8 \%$ were receiving psychotherapy over the previous 12 months, and $21.8 \%$ had received at least one of these treatments.[11] Eisenberg recorded that the number one prescribed medications were antidepressants, prescribed by primary care physicians among $48.2 \%$ and by psychiatrists for $35.2 \%$. He also records that $15.8 \%$ of students with mental health issues reported that they engaged in neither professional nor non-professional treatment. According to the Jed Foundation, stigma is the number one reason why students do not seek out help on campuses, specifically embarrassment.[12] They stated that only 23\% of students surveyed in 2006 would feel comfortable with a close friend knowing about the mental health concerns. The next most prevalent reason reported were distrust of confidentiality, finances, and the fear that accepting that they are struggling would mean that they are incapable of leading healthy, productive lives. This causes students to be reclusive when their mental health is concerned and reinforces the stigma.

The part of Eisenberg's study that is worrisome for other reasons altogether is that among those students taking medications, $14.7 \%$ were doing so without a prescription.[11] Self-medication and substance misuse have become a part of what seems to now be tolerated in regard to college culture, even being seen as an expected behavior in a ritualistic, coming-of-age sense. Greek life is highlighted in a study done by Harvard University, cited by Addiction Center. This study claims that 4 out of 5 members of fraternities and sororities are binge drinkers, compared to only 2 out of 5 of students who were not members of fraternities or sororities.[13] In fact, $62 \%$ of sorority women admitted to heavy drinking, compared to only $41 \%$ of non-sorority affiliated college women. The main causes for this, cited by the same article, are group living, lack of supervision, hazing and peer pressure. The Addiction Center discusses why students turn to alcohol and drugs as means of coping. The main reasons highlighted in the article, College Drug Abuse, are cited as stress, course load, curiosity and peer pressure. Alcohol, marijuana, Adderall, and ecstasy are quoted as being the top substances that are misused by college students, with alcohol being the number one due to its accessibility and acceptance as part of the culture. They write about alcohol:

Alcohol is the most popular and dangerous drug on college campuses by far. To many, drinking is synonymous with the college experience; alcohol is nearly always present at house parties, sporting events and student get-togethers. Because the use of alcohol during college is widespread and 
often condoned, many college students end up drinking more alcohol more frequently than their peers who aren't in college.[13]

Alcohol tends to lead to legal complications, aside from the major health concerns it presents. Results are fights, drunk driving arrests, and ruined relationships on top of poor academic performances that sometimes result in expulsion, loss of financial aid, or simply dropping out of the institution. However, it is common for students to equate assimilation into the college culture with acceptance and personal worth in the eyes of their peers. When they are suffering from depression and anxiety with security needs not being met, perhaps it is more attractive to suffer within an illusion of acceptance and belonging than to go it alone. American Addiction Centers cites 1,825 college students 18-24 years of age die from alcohol-related injuries, 696,000 students of the same demographic are assaulted by a student who has been drinking, 1 in 4 students report problems with academic performance as a result of their drinking, and 97,000 students18-24 years of age state they have been sexually assaulted or raped due to alcohol-related circumstances. Dr. Eric Wood, who is interviewed in this same article, discusses the potential for increased correlation between alcohol or substance misuse and prior pre-existing depression and anxiety.

We know that as compared to prior generation, these students (referring to the 2019 entering freshman class) will have more prior history with mental health issues (depression and anxiety), will have a lower age of when they first tried alcohol or drugs, be more likely to have been prescribed psychotropic medication, and will likely know someone who has either been sexually assaulted or attempted suicide. . . and all of this prior to starting college.[13]

Dr.Woods' statement suggests that counseling center case loads are going to continue to skyrocket due to earlier experiences of substance misuse and mental health concerns, which, according to the current statistics, mean that what universities are currently experiencing will simply be significantly exacerbated once the college culture comes into play with these individuals. Dr. Wood continues on to make an extraordinary point that the number of students who do not drink at all during college is increasing, therefore, studying them could produce some insight into preventive measures that could be exploited by administrations.

Stigma is a major concern for students dealing with mental health disorders, but it extends beyond the individual to the community as a whole. Jessica Wolf cites Michael Gaddis, "in places where their peers are stigmatizing mental health treatment, students do not want to even acknowledge their mental health struggles." [14] When students feel embarrassed by their mental health conditions, they turn from the idea of seeking treatment, further cementing the effects of their disorder and the impairment of academic and social performance.
One of the ways that UCLA battled this was by initiating a voluntary screening for incoming freshman in 2017 to catch students early on before symptoms could overrun them. This program was called the Depression Grand Challenge. "Those whose results showed they were at risk of suicide, severe depression or other serious mental health problems were offered access to treatment at UCLA."[14]

Suicide ideation has been correlated to severe depression in students and especially among those not seeking treatment. The PHQ- 9 was utilized during a study performed by Emory University as part of their American Foundation for Suicide Prevention-sponsored College Screening Project. Through their investigation, their findings revealed that out of 729 students surveyed over a three year period, $11.1 \%$ endorsed current suicidal ideation within the past four weeks and $16.5 \%$ had a suicide attempt of episode of self-harm during their lifetime.[15] Depression rates were significantly higher with students who endorsed current suicide ideation than those who did not endorse current ideation. $28.5 \%$ of students with high PHQ-9 scores (15 or higher) reported suicidal ideation. Furthermore, their study revealed that $85 \%$ of students who showed moderate to severe symptoms of depression were not receiving any form of treatment.[15] This further proves that these issues are real and are going undiagnosed and untreated by universities.

\section{Future Directions}

Anxiety, depression, and substance misuse issues and the tragedies that are associated with them are all preventable. University counseling centers are understaffed and overwhelmed with the number of students that each counselor is responsible for managing. These studies show that funding is a major contributor to the lack of staff in these centers. With the rising need for mental health treatment for students, funding must be made available. The affordability of mental health counselors for university counseling centers is not something that universities can afford to look the other way on any longer. School shootings, falling retention rates, and mental health issues across the board can be prevented and should be prevented to the best of every university's ability.

Faculty training programs are a good motivator for finding the funding required to bring on more counselors so that they can be trained appropriately on how to recognize the symptoms in students when those symptoms present themselves. It is not just for the individual's sake, but for the campus' sake as well. We are seeing a rise in school shootings and it is stated by Allison Paolini that $78 \%$ of school attackers have a history of suicidal attempts or ideation, although only $17 \%$ were diagnosed at dealing with a mental health disorder.[16] Those numbers imply that suicide ideation had been present, which also implies a mental health disorder, but treatment was not prevalent in these individuals. Had treatment been engaged, these shootings may not have happened. This is what it means when people are "slipping through the cracks".

Another direction that needs to be taken is not just training 
faculty members, but resident advisors in dormitories as well. Faculty have classroom interaction with students, which is around three hours a week. This time is usually spent teaching and not actually interacting in a social sense that could produce a glimpse at underlying symptoms a student may be dealing with. But resident advisors, especially when freshmen are concerned, do room checks, manage curfews (where applicable), have hall meetings with the residents, and see the students residing in these dormitories on a regular basis. Training is of utmost importance for these positions and may even cause universities to rethink how they hire resident advisors. This also applies to hall directors, front desk workers, and any other campus-employed individual that has a daily interaction with student residents. This is where the majority of symptoms could be noticed before overrunning a student's life. It should be a responsibility for those managing the halls and floors of these student residents to have an appropriate understanding of symptoms to be able to target those showing potential warning signs and they must also understand how to apply said knowledge in order to intervene in an appropriate and timely manner. These are the potentially the only employed persons on campuses that have the ability to find out whether or not a student is not leaving their dorm or is routinely alone. Furthermore, screenings should be implemented on a voluntary basis to incoming students who plan to live on campuses. This way, campuses have a baseline understanding of what the incoming students have been through. The PHQ9 would suffice until universities find something that works more efficiently for their specific community of students.

Currently, universities are not effective in their treatment options and methods, hands tied or not. While many universities have a number of resources available, these resources are spread too thinly to reach the majority of the population and are often only effective to that minority population when sought out. For those students who receive the treatment, however, these studies show that they make a difference. If these treatment options are making a difference for the students that they reach, then these treatment options should be far more significantly funded in order to increase outreach and to penetrate these mental health crises to the core. This is the only way that we will see declines in issues such as suicide, violence, substance misuse, and drop-out rates.

\section{Conclusion}

While universities are at a significant disadvantage for addressing mental health needs of post-secondary students, they do have the ability to make significant changes as well in this aspect of college culture. The studies that have been addressed in this paper were sampling real college populations, many of them being conducted by the university whose student body's population they represented (Emory University, Texas Christian University, UCLA etc.). It is within the power of the universities to analyze their own populations and to train their faculty and staff members to recognize what is happening within these populations. It is also within their power to educate their students on the presence of counseling and treatment options that are available on campus. When students are unaware of services that are offered, the university has failed them. It is my belief that funding needs to be found, training needs to happen, and counseling centers must be staffed in order for these issues to ever have a hope of being resolved. Beyond this, universities need to encourage and assist in the transition period for students. It is quite reasonable to assume that a student who never finds their place among the student body will be more likely to go through their college career relatively alone and unnoticed, due to the nature of cliques in school environments. This is something that should be studied further on a campus by campus basis.

\section{Author Biography}

Jordan Talley graduated in May 2018 with a Bachelor of Science in Integrative Studies. While attending the University of North Texas, Jordan was involved in the Honors College and campus-based recovery programs, gaining an interest in student mental health issues. Jordan is from Grapevine, TX and plans to pursue a career in Higher Education Administration and to continue his research into campus-based recovery and support programs for students.

\section{References}

${ }^{[1]}$ L. Winerman. By the numbers: Stress on campus. Monitor on Psychology, 48(8), 2017.

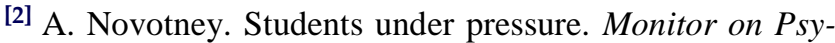
chology, 48(8), 2017.

[3] Dastan T Faeq. Depression among students: Critical review.

[4] American Psychiatric Association. Diagnostic and Statistical Manual of Mental Disorders. Arlington, VA, 2013.

[5] Sakurako Chako Mori. Addressing the mental health concerns of international students. Journal of counseling \& development, 78(2):137-144, 2000.

https://doi.org/10.1002/j.1556-6676.2000.tb02571.x

[6] S.A. McLeod. Maslow's hierarchy of needs. WWW . simplypsychology.org/maslow.html. Accessed: 2017-09-16.

${ }^{[7]}$ Karin F Brockelman and Anna M Scheyett. Faculty perceptions of accommodations, strategies, and psychiatric advance directives for university students with mental illnesses. Psychiatric rehabilitation journal, 38(4):342, 2015.

https://psycnet.apa.org/doi/10.1037/prj0000143

${ }^{[8]}$ Steven Bushong. College counseling centers remain understaffed though demand is strong, survey finds. The Chronicle of Higher Education, 2009.

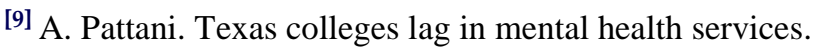
https://www. texastribune.org/2016/08/ 09/texas-universities-struggle. Accessed: 2018-04-15. 
[10] International Association of Counseling Services. Statement regarding recommended staff to student ratios. http://www.iacsinc.org/ staff-to-student-ratios.html. Accessed: 2018-03-22.

[11] Daniel Eisenberg, Justin Hunt, Nicole Speer, and Kara Zivin. Mental health service utilization among college students in the United States. The Journal of nervous and mental disease, 199(5):301-308, 2011.

doi: 10.1097/NMD.0b013e3182175123

${ }^{\text {[12] }}$ The Jed Foundation. Depression and anxiety among college students. https: / / www . jedfoundation. org. Accessed: 2018-03-14.

[13] Addiction Center. Drinking and drug abuse in greek life. https://www.addictioncenter.com/ college/. Accessed: 2018-04-08.

[14] J Wolfe. Study shows stigma around mental health on campus correlates with students not seeking treatment. http://newsroom.ucla.edu/releases/. Accessed: 2018-04-12.

[15] Steven J Garlow, Jill Rosenberg, J David Moore, Ann P Haas, Bethany Koestner, Herbert Hendin, and Charles B Nemeroff. Depression, desperation, and suicidal ideation in college students: results from the American Foundation for Suicide Prevention college screening project at Emory University. Depression and anxiety, 25(6):482-488, 2008.

https://doi.org/10.1002/da.20321

[16] Allison Paolini. Cyberbullying: role of the school counselor in mitigating the silent killer epidemic. International Journal of Educational Technology, 5(1):1-8, 2018. 